\title{
Ácido úrico y síndrome metabólico en una población masculina de pilotos de líneas aéreas
}

\author{
Uric acid and metabolic syndrome in aircrew members population
}

\author{
Juan Medina Font', César Alonso Rodríguez', Carlos Gutiérrez Ortega² ${ }^{2}$ Pilar Montenegro Álvarez de Tejera ${ }^{3}$, \\ Javier Gómez de los Terreros Sánchez ${ }^{4}$ \\ 1. Centro de Instrucción de Medicina Aeroespacial. Ministerio de Defensa. Madrid. España. \\ 2. Servicio de Medicina Preventiva. Hospital Central de la Defensa. Madrid. España. \\ 3. Servicio de Farmacia Clínica. Farmacocinética. Hospital Central de la Defensa. Madrid. España. \\ 4. Universidad Complutense de Madrid. Facultad de Medicina. Madrid. España.
}

Recibido: 28-10-11

Aceptado: 03-01-12

\section{Correspondencia}

Juan Medina Font

Centro de Instrucción de Medicina Aeroespacial. Ministerio de Defensa.

Arturo Soria, 82

28027 Madrid. España

Tfno: 606393494/914101791

E-mail: jmedfon@ oc.mde.es

Resumen

Introducción: Ácido úrico sérico y Síndrome Metabólico, son factores que se relacionan fuertemente con enfermedades cardiovasculares. Objetivo: conocer la prevalencia de Síndrome Metabólico así como la concentración de Ácido úrico sérico en una población aeronáutica y determinar qué posibles relaciones muestran unos niveles normales y/o patológicos de Ácido úrico con respecto a la presencia o ausencia de Síndrome Metabólico.

Material y métodos: Población de 790 pilotos de líneas aéreas, todos varones. Se siguió el criterio del National Cholesterol Education Program para el diagnóstico de Síndrome Metabólico, pero modificado, utilizándose en lugar de circunferencia cintura el índice de masa corporal.

Resultados: El 25,4\% de los pilotos presentaron valores elevados de Ácido Úrico. El 19,9\% de los pilotos presentó Síndrome Metabólico. El riesgo de presentar Síndrome Metabólico por tener el Ácido úrico elevado frente al riesgo que presentaron los que tienen el Ácido úrico normal (razón de prevalencias) fue de 2,6 con un intervalo de confianza al 95\% entre 2 y 3,4 y con una significación de p<0,001. Existe una asociación lineal positiva entre el aumento de la concentración de Ácido úrico y el número de factores diagnósticos positivos de Síndrome Metabólico ( $\mathrm{p}<0,001)$.

Conclusiones: Nuestros resultados sugieren que la hiperuricemia puede ser una complicación relacionada son el propio Síndrome Metabólico; la obesidad abdominal puede condicionar un aumento en la concentración de Ácido úrico. Se hace preciso aumentar los estudios sobre poblaciones aeronáuticas respecto a estos factores metabólicos con objeto de aumentar la seguridad en vuelo.

Med Segur Trab (Internet) 2012; 58 (226) 49-58

Palabras clave: pilotos, ácido úrico, síndrome metabólico. 


\section{Abstract}

Fundamentals: Uric acid and metabolic syndrome are strongly related to cardiovascular disease. Objective: To assess the prevalence of metabolic syndrome as well as the levels of uric acid among aircrew members and to determine the relationship between the levels of uric acid and the prevalence of metabolic syndrome.

Methods: We studied a population of 790 male airline pilots. We used the definition of metabolic syndrome of the National Cholesterol Education Program Adult Treatment Panel III, but modified by replacing waist circumference for the measurement of body mass index.

Results: $25.4 \%$ of the pilots presented elevated level of uric acid. $19.9 \%$ of pilots presented metabolic syndrome. The risk to present metabolic syndrome in pilots with uric acid elevated in relation to those pilots with normal uric acid was 2.6 higher with a confidence interval of 95\% between 2 and 3,4 with a significance of $\mathrm{p}<0.001$. There is a positive linear association between the increased concentration of uric acid and the number of metabolic syndrome diagnostic criteria $(\mathrm{p}<0,001)$.

Conclusions: Our results suggest that hyperuricemia can be a complication related to the metabolic syndrome. The abdominal obesity may condition an increased concentration of serum uric acid. It is necessary to increase the number of studies on aircrew members in relation to the above mentioned metabolic factors in order to increase the flight safety.

Med Segur Trab (Internet) 2012; 58 (226) 49-58

Key words: aircrew members, uric acid, metabolic syndrome. 


\section{INTRODUCCIÓN}

El ácido úrico sérico (AUS) elevado se asocia a enfermedades cardiovasculares como el infarto de miocardio ${ }^{1}$, accidente vascular cerebral, hipertensión e insuficiencia cardíaca ${ }^{2}$; esta asociación es menor si se trata de sujetos que presentan una concentración de AUS que se sitúa en el tercio inferior al rango habitualmente establecido como fisiológico ${ }^{3}$. No obstante, un estatus de patología cardiovascular, suele ser el resultado del concurso aislado o colectivo de numerosos factores de riesgo que tienen probada responsabilidad en la etiopatogenia del proceso. En los últimos años se ha definido una entidad clínica, el Síndrome Metabólico (SM), que agrupa una parte relevante de estos factores, que predisponen al desarrollo de enfermedad cardiovascular. Por tanto, AUS y SM, son dos factores que se relacionan fuertemente con enfermedades cardiovasculares.

El estudio propuesto, se ha desarrollado en un colectivo profesional que presenta importantes peculiaridades en lo que respecta a su estilo de vida. Al tratarse de pilotos de líneas aéreas, están sometidos a unos horarios laborales desiguales en cuanto a su duración, a cambios ambientales provocados por desplazamientos a través de diferentes husos horarios que alteran el ritmo circadiano, así como a unos hábitos alimenticios condicionados laboralmente y que, en general, no se adaptan a los estándares nutricionales y dietéticos. Otro factor a tener en cuenta es la situación de fuerte sedentarismo laboral, patente sobre todo en desplazamientos aéreos de larga duración. Todas estas características y hábitos derivados del ejercicio profesional, muestran un colectivo laboral en el que, podrían estar acentuados diversos factores de riesgo cardiovascular.

Tras estas consideraciones, nos proponemos conocer cuál es la situación en la que se encuentra el personal aeronáutico que pasa reconocimiento psicofísico en nuestro centro, con respecto a la prevalencia de SM y a las concentraciones séricas de AUS, siendo útil determinar las posibles relaciones entre unos valores normales o patológicos de AUS con relación a la presencia o ausencia de SM. También comprobaremos cómo varían las concentraciones de AUS en función del número de factores diagnóstico positivos de SM en el personal de vuelo estudiado.

\section{SUJETOS Y MÉTODO}

Población de 790 pilotos de líneas aéreas, que pasaron reconocimiento psicofísico en el Centro de Instrucción de Medicina Aeroespacial (CIMA) para renovar su licencia de vuelo. El grupo estuvo formado en su totalidad por varones, con una edad media de $41,86 \pm 10,05$ años. El protocolo de recogida de datos se llevó a cabo a partir de un cuestionario clínico-epidemiológico de carácter voluntario y confidencial, tras muestreo aleatorio. El $2 \%$ de los sujetos denegaron su participación; las muestras biológicas se procesaron siguiendo una metodología estándar para su obtención, conservación y análisis.

Para el diagnóstico de SM, se siguió el criterio del National Cholesterol Education Program (NCEP) ATPIII ${ }^{4}$, pero modificado al no considerarse la circunferencia cintura (CC) como uno de los criterios diagnóstico, utilizándose en su lugar el índice de masa corporal (IMC), quedando el cuadro de inclusiones de la siguiente forma.

Para el diagnóstico de SM en varones, deben de ser positivos, al menos tres de los siguientes cinco factores:

- IMC $\geq 25$.

- Tensión arterial: positivo si PAS $\geq 130 \mathrm{~mm}$ de $\mathrm{Hg}$ y/o PAD $\geq 85 \mathrm{~mm}$ de $\mathrm{Hg}$ y/o si está tomando medicación antihipertensiva.

- HDL-C: positivo $s i<40 \mathrm{mg} / \mathrm{dL}$.

- Triglicéridos: positivo $s i \geq 150 \mathrm{mg} / \mathrm{dL}$.

— Glucosa: positivo $s i \geq 100 \mathrm{mg} / \mathrm{dL}$. 
El AUS sérico se determinó colorimétricamente mediante el método de la uricasa en autoanalizador Hitachi 917 en suero procedente de muestras de sangre total extraídas en el CIMA tras ayuno de 12 horas. Se consideraron sujetos normouricémicos, los que presentaron valores iguales o inferiores a $7 \mathrm{mg} / \mathrm{dL}$, e hiperuricémicos los que mostraron valores superiores a dicha concentración.

En lo referente a la metodología estadística, como índices de tendencia central y de dispersión se emplearon: para las variables cuantitativas, la media aritmética y la desviación estándar; y para las categóricas su frecuencia relativa en tantos por ciento. La estimación del riesgo de SM por tener el AUS elevado frente a los que lo tienen normal, se estableció mediante la razón de prevalencias y la prueba exacta de Fisher. Mediante el test de Pearson se determinó la correlación lineal entre variables cuantitativas. Se consideró como estadísticamente significativo un valor de la $\mathrm{p}<0,05$. Por último, se procedió a un análisis estadístico de comparaciones múltiples (Test de Bonferroni), en función del número de factores diagnósticos de SM, utilizando como variable dependiente el AUS. Para todo ello se utilizaron los paquetes estadísticos SPSS v 15 y Epidat v 3.1.

\section{RESULTADOS}

El 74,6\% de la población presentó valores normales de AUS frente al 25,4\% que lo tuvo elevado.

El diagnóstico de SM, es decir, aquéllos que presentaron tres o más factores diagnósticos positivos estuvo presente en el 19,9\% de la población, frente al 80,1\% sin SM.

Los pilotos con valores normales de AUS mostraron una prevalencia de SM del 14,1\% (Figura n. ${ }^{\circ}$ ), mientras que los pilotos en situación hiperuricémica presentaron SM en un $36,8 \%$ (Figura n. $^{\circ}$ 2).

Figura n. $^{\circ}$ 1. Frecuencias relativas $(\%)$ de S.M. en normouricémicos

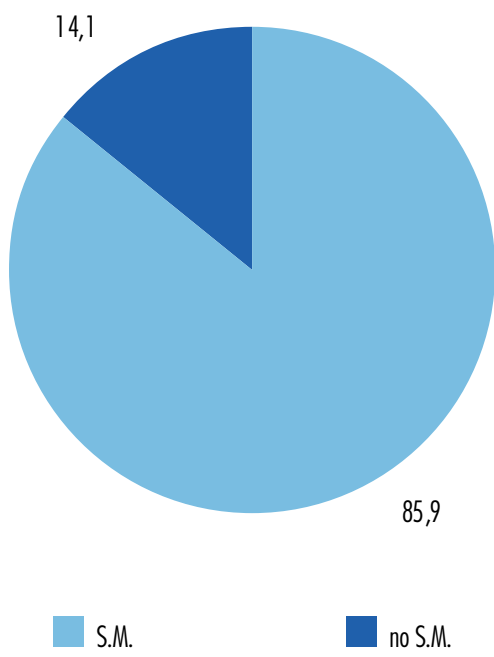

En los pilotos con AUS elevado, el IMC (66,2\%) y la tensión arterial $(52,7 \%)$ fueron los dos factores diagnóstico positivo que mostraron una mayor frecuencia de aparición, seguidos de triglicéridos (40,8\%), glucosa $(30,8 \%)$ y HDL-C $(11,9 \%)$. 
Figura $n^{\circ}$ 2. Frecuencias relativas $(\%)$ de S.M. en hiperuricémicos

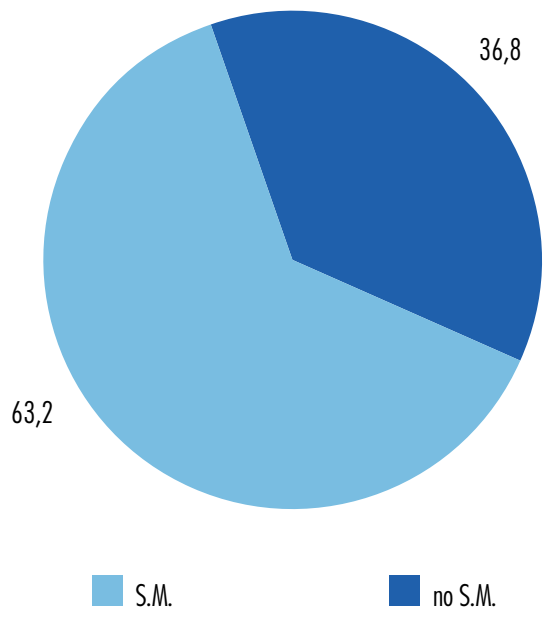

El riesgo de padecer SM es 2,6 veces superior en los individuos con uricemias mayores de $7 \mathrm{mg} / \mathrm{dL}$ (IC 95\%: 2 a 3,4 veces) que en el grupo con concentraciones inferiores a dicha cantidad $(\mathrm{p}<0,001)$. De los primeros la padece un $36,8 \%$, mientras que de los últimos lo hace un $14,1 \%$. (Tabla I).

Tabla I. Distribución de valores normales y elevados de A.U y de presencia o ausencia de S.M. en la muestra de pilotos de líneas aéreas.

\begin{tabular}{cccc}
\hline & S.M. & No S.M. & Total \\
\hline UA $>7 \mathrm{mg} / \mathrm{dL}$ & $36,8 \%(74 / 201)^{*}$ & $63,2 \%(127 / 201)$ & 201 \\
$\mathrm{UA} \leq 7 \mathrm{mg} / \mathrm{dL}$ & $14,1 \%(83 / 589)$ & $85,9 \%(506 / 589)$ & 589 \\
\hline Total & 157 & 633 & 790 \\
\hline
\end{tabular}

* RP: 2,6 (IC95\%(2-3,4); p<0,001

El porcentaje de sujetos que presentaban como máximo un factor diagnóstico de SM, fue mayor en el grupo con valores de AUS normal que en el que presenta AUS elevado; sin embargo, cuando se consideran dos o más factores diagnóstico, el porcentaje se invierte, siendo mayor en el grupo de sujetos con elevado AUS que en el que tiene un AUS normal (Tabla II).

Tabla II. Porcentaje de pilotos normouricémicos e hiperuricémicos en función del número de factores diagnóstico de S.M.

\begin{tabular}{ccc}
\hline N. $^{\text { de factores diagnósticos de S.M.* }}$ & $\begin{array}{c}\text { Normouricemia } \\
(\text { A.U. } \leq 7 \mathrm{mg} / \mathrm{dL})\end{array}$ & $\begin{array}{c}\text { Hiperuricemia } \\
\text { (A.U. >7 mg/dL) }\end{array}$ \\
\hline 0 & $29,7 \%(175 / 589)$ & $12,4 \%(25 / 201)$ \\
1 & $32,9 \%(194 / 589)$ & $24,4 \%(49 / 201)$ \\
2 & $23,3 \%(137 / 589)$ & $26,4 \%(53 / 201)$ \\
No SM & $\mathbf{8 5 , 9 \% ( 5 0 6 / 5 8 9 )}$ & $\mathbf{6 3 , 2 \% ( 1 2 7 / 2 0 1 )}$ \\
3 & $11,0 \%(65 / 589)$ & $23,9 \%(48 / 201)$ \\
4 & $2,9 \%(17 / 589)$ & $10,9 \%(22 / 201)$ \\
5 & $0,2 \%(1 / 589)$ & $2,0 \%(4 / 201)$ \\
\hline SM & $\mathbf{1 4 , 1 \% ( 8 3 / 5 8 9 )}$ & $\mathbf{3 6 , 8} \%(\mathbf{7 4} / \mathbf{2 0 1})$ \\
\hline
\end{tabular}

$*(0,1,2)=$ no S.M.; $(3,4,5)=$ S.M.

El AUS se eleva según vamos considerando en la población un aumento del número de factores diagnóstico de SM positivos (Figura $\mathrm{n}^{\circ} 3$ ); de hecho existe una asociación 
lineal positiva entre el aumento de la concentración de AUS y el número de factores diagnóstico positivos de SM $(\mathrm{p}<0,001)$.

Figura n. ${ }^{\circ}$ 3. Concentración de ácido úrico respecto al número de factores diagnóstico positivos de S.M. $(p<0,001)$

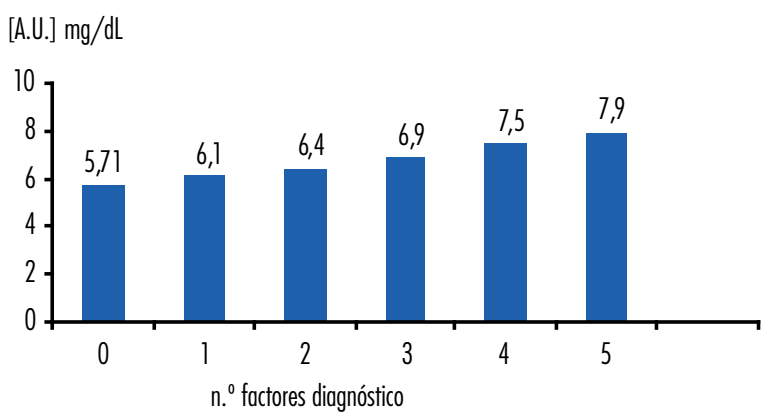

El análisis estadístico de comparaciones múltiples (Test de Bonferroni) se aplicó al número de factores diagnósticos de SM, utilizando como variable dependiente el AUS; los resultados fueron los siguientes (Tabla III):

Tabla III. Comparaciones múltiples entre el $\mathbf{n} .^{\circ}$ de factores diagnóstico de SM, usando el AUS como variable dependiente

Variable dependiente: AUS

Bonferroni

\begin{tabular}{|c|c|c|c|c|c|}
\hline \multirow{2}{*}{ (I) N..$^{\circ}$ FACTORES } & \multirow{2}{*}{ (J) N. ${ }^{\circ}$ FACTORES } & \multirow{2}{*}{$\begin{array}{c}\text { Diferencia de } \\
\text { medias (I-J) }\end{array}$} & \multirow{2}{*}{ Sig. } & \multicolumn{2}{|c|}{ Intervalo de confianza al 95\% } \\
\hline & & & & Límite superior & Límite inferior \\
\hline \multirow[t]{5}{*}{0} & 1 & $-.3854(*)$ & ,010 & -.72 & -.05 \\
\hline & 2 & $-.6506(*)$ &, 000 & -1 & -.3 \\
\hline & 3 & $-1.1417\left(^{*}\right)$ &, 000 & -1.6 & -.7 \\
\hline & 4 & $-1.8408\left(^{*}\right)$ &, 000 & -2.5 & -1.2 \\
\hline & 5 & $-2.2295\left(^{*}\right)$ & 001 & -3.8 & -.7 \\
\hline \multirow[t]{4}{*}{1} & 2 & -.2652 &, 312 & -.6 & .07 \\
\hline & 3 & $-.7563(*)$ &, 000 & -1.15 & -.36 \\
\hline & 4 & $-1.4554(*)$ &, 000 & -2.1 & -.86 \\
\hline & 5 & $-1.8441\left(^{*}\right)$ & 009 & -3.4 & -.27 \\
\hline \multirow[t]{3}{*}{2} & 3 & $-.4912(*)$ & 007 & -.9 & -.08 \\
\hline & 4 & $-1.1902(*)$ &, 000 & -1.8 & -.6 \\
\hline & 5 & $-1.5789\left(^{*}\right)$ &, 049 & -3.2 & -.002 \\
\hline \multirow[t]{2}{*}{3} & 4 & $-.6991\left(^{*}\right)$ & ,023 & -1.3 & -.05 \\
\hline & 5 & -1.0878 & ,665 & -2.7 & .5 \\
\hline 4 & 5 & -.3887 & 1,000 & -2.0 & 1.3 \\
\hline
\end{tabular}

* La diferencia de medias es significativa al nivel $<0,05$.

1. Los que no presentan algún factor tienen un AUS menor que los que presentan al menos uno. Así pues, respecto a los que no presentan ningún factor:

a. Los que tienen un factor presentan un AUS superior entre 0,05 y 0,72 $\mathrm{mg} / \mathrm{dL}$ $(\mathrm{p}=0,01)$

b. Los que tienen dos factores presentan un AUS superior entre 0,3 y $1 \mathrm{mg} / \mathrm{dL}$ $(\mathrm{p}<0,001)$ 
c. Los que tienen tres factores presentan un AUS superior entre 0,7 y $1,6 \mathrm{mg} / \mathrm{dL}$ $(\mathrm{p}<0,001)$

d. Los que tienen cuatro factores presentan un AUS superior entre 1,2 y 2,5 mg/ $\mathrm{dL}(\mathrm{p}<0,001)$

e. Los que tienen los cinco factores presentan un AUS superior entre 0,7 y 3,8 $\mathrm{mg} / \mathrm{dL}(\mathrm{p}=0,001)$.

2. No se encuentran diferencias significativas de AUS entre los que presentan un factor y los que muestran dos $(\mathrm{p}=0,312)$. Sin embargo, si se ve incrementado significativamente el AUS respecto de los que tienen un factor en:

a. 0,36 a $1,15 \mathrm{mg} / \mathrm{dL}$ en los que tienen tres factores $(\mathrm{p}<0,001)$

b. 0,86 a $2,1 \mathrm{mg} / \mathrm{dL}$ en los que tienen cuatro factores $(\mathrm{p}<0,001)$

c. 0,27 a $3,4 \mathrm{mg} / \mathrm{dL}$ en los que tienen cinco factores $(\mathrm{p}=0,009)$. Nótese nuevamente la gran longitud de este último intervalo de confianza, reducible sin duda con un tamaño de muestra adecuado.

3. Se observa un incremento estadísticamente significativo de AUS entre los que muestran tres o más factores y los que solo muestran dos. Así:

a. Con tres factores aumenta de 0,08 a $0,9 \mathrm{mg} / \mathrm{dL}(\mathrm{p}=0,007)$

b. Con cuatro factores aumenta de 0,6 a $1,8 \mathrm{mg} / \mathrm{dL}(\mathrm{p}<0,001)$

c. Con cinco factores de 0,002 a $3,2 \mathrm{mg} / \mathrm{dL}(\mathrm{p}=0,049)$. No se puede considerar esta diferencia estadísticamente significativa aunque $\mathrm{p}<0,05$. Biológicamente este intervalo de confianza es excesivamente amplio.

4. Los que presentan tres factores tienen un AUS menor (de 0,05 a 1,3 mg/dL) que los que tienen cuatro factores $(\mathrm{p}=0,023)$.

5. No se encuentra AUS estadísticamente diferentes entre los que poseen tres o más factores.

\section{DISCUSIÓN}

En este estudio se ha considerado el IMC como uno de los criterios de inclusión para el diagnóstico de SM, en lugar de la circunferencia de la cintura que es el criterio que propone el NCEP ATP III. Hay diversos estudios que reconocen una similitud de resultados en la prevalencia de SM usando indistintamente IMC o CC; así se han correlacionado ambas medidas en análisis sobre muestras grandes 5 . Valores medios de prevalencia de SM en estudios que utilizan la CC, son similares a otros que usan el IMC; este último ya es utilizado en lugar del perímetro de la cintura como criterio de inclusión de SM en otros estudios como en el PROCAM (Prospective Cardiovascular Münster) en el que se reclutaron 19.698 personas en el Noroeste de Alemania, encontrando una prevalencia del 17,1\% en hombres ${ }^{6}$. La Asociación Americana de endocrinólogos (AACE), utiliza como criterio diagnóstico de resistencia insulínica, un IMC superior a 25 es decir un criterio de sobrepeso, en lugar de usar la circunferencia de la cintura ${ }^{7}$, lo que concuerda con el Grupo Europeo para el Estudio de la Resistencia Insulínica (EGIR) que admite una correlación equivalente entre la resistencia insulínica y la obesidad, al usar el cociente cintura/cadera o el IMC $^{8}$. De cualquier forma, la técnica de medir la circunferencia de la cintura varía con respecto a distintos protocolos, es compleja y de difícil reproducibilidad ${ }^{9}$ y sus límites deberían variar en función del grupo étnico estudiado, consideración que ya es recogida en la definición de la IDF (Internacional Diabetes Federation).

Diversos trastornos metabólicos se asocian con la vida sedentaria, el envejecimiento y la obesidad central, e inducen alteraciones en los niveles de presión arterial, lípidos y glucosa aumentando el riesgo de eventos cardiovasculares, además de predisponer al aumento de factores diagnóstico positivos del SM. No obstante, hay otros posibles factores predisponentes potenciales a un aumento de sujetos con SM; así, parece que la hiperuricemia puede ser una complicación derivada del propio $\mathrm{SM}^{10}$; al parecer, la 
obesidad abdominal puede condicionar un aumento en la concentración de AUS ${ }^{11}$. Para algunos autores, la hiperuricemia es considerada una característica del SM a pesar de que el AUS no sea considerado un criterio diagnóstico de SM, entre otras cosas porque la alteración fisiopatológica que desencadena el aumento de AUS no es conocida en su totalidad $^{12}$.

Se discute actualmente si el aumento del AUS implica un riesgo independiente de enfermedad vascular en sujetos con SM; sí se ha comprobado que dicha relación existe en sujetos que no presentan SM; sin embargo puede que dicho aumento de AUS no esté independientemente asociado a eventos cardiovasculares en pacientes con $\mathrm{SM}^{13}$.

Se ha comprobado que la hiperuricemia y el SM son factores de riesgo independientes para desarrollar resistencia insulínica en mujeres, sin embargo en hombres sí se mantiene asociado a resistencia insulínica el S.M., pero no así la hiperuricemia ${ }^{14}$. En otros trabajos, en obesos no diabéticos, el riesgo de disfunción renal es independiente de la presencia o ausencia de $\mathrm{SM}^{15}$.

Los resultados que hemos obtenido sobre la mayor prevalencia de SM en sujetos hiperuricémicos que en normouricémicos, son equivalentes a los obtenidos por LópezSuárez et al $^{12}$, quiénes tras análisis de regresión múltiple comprobaron cómo sexo y SM se encontraban asociados independientemente con el AUS. Sin embargo, la ausencia de estudios descriptivos en poblaciones de pilotos de líneas aéreas o personal aeronáutico asimilable nos imposibilita discutir posibles implicaciones de naturaleza laboral y ambiental.

Aunque hemos constatado la existencia de una asociación lineal positiva entre el aumento de la concentración de AUS y el número de factores diagnósticos positivos de SM $(\mathrm{p}<0,001)$, hemos querido completar el estudio con un análisis de comparaciones múltiples que se refleja en la tabla III, sobre la cuál tenemos que hacer una serie de precisiones:

1. Los que tienen los cinco factores frente a los que no presentan algún factor tienen un AUS superior entre 0,7 y $3,8 \mathrm{mg} / \mathrm{dL}(\mathrm{p}=0,001)$. Aunque se observó este incremento como muy significativo hay que destacar la gran amplitud del intervalo de confianza debido, muy posiblemente, al reducido número de los pilotos con cinco factores del SM $(n=5)$. Habría pues que incrementar el tamaño de muestra del grupo para poder reducir ese intervalo de confianza.

2. No se encuentran diferencias estadísticamente significativas entre la uricemia de los pilotos con un factor diagnóstico positivo y la de los que presentan dos factores diagnóstico positivos $(\mathrm{p}=0,312)$, aunque sí se produce un aumento de AUS en este último grupo.

3. No se encuentran concentraciones de AUS significativamente diferentes entre los que poseen tres o más factores diagnóstico, en este caso con toda probabilidad debido a los amplios intervalos de confianza, que se podrían reducir con unos tamaños de muestra adecuados.

En la Gráfica n. ${ }^{\circ}$ 1, se pone de manifiesto la semejanza de las dispersiones de los datos entre los que no tienen factores del SM hasta los que tienen tres. Se observa una relación lineal positiva. Con cuatro, y sobre todo con cinco factores del SM, las dispersiones aumentan lo que hace perder la significación estadística. 


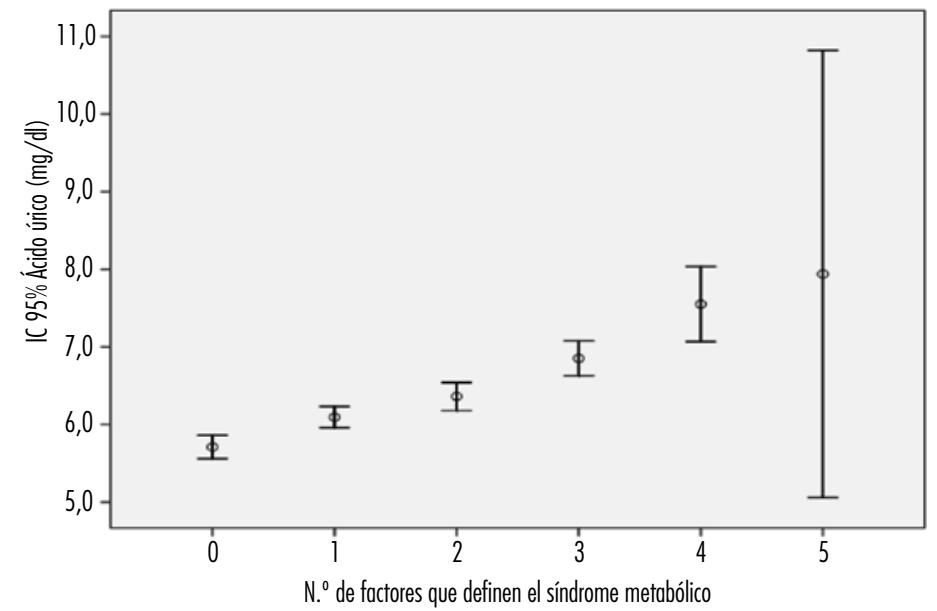

Hay que destacar la práctica imposibilidad de incrementar el tamaño de muestra de estos dos últimos grupos dada la idiosincrasia de la población estudiada; esta limitación metodológica se justifica al tratarse de una población sometida a unos estándares de salud obligados para la renovación de su licencia de vuelo.

En el diagrama de cajas que se muestra en la Gráfica $n .^{\circ}$ 2, se representan con líneas horizontales dentro de las cajas $\left(\mathrm{P}_{25}\right.$ y $\left.\mathrm{P}_{75}\right)$ las medianas. Se observa su claro incremento hasta los tres factores; también se constata cómo en las correspondientes a cuatro y cinco factores se pierde la normalidad de las distribuciones (las medianas no dividen en dos partes iguales a las cajas).

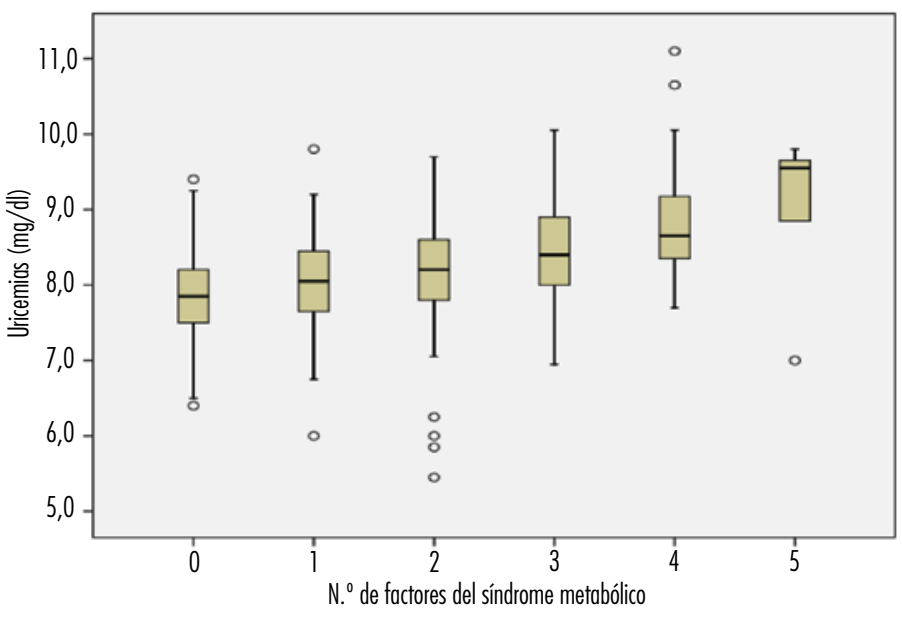

Aunque hay una abundante bibliografía que nos informa sobre las prevalencias, tanto de SM como de los niveles de AUS, la mayoría de los autores se refieren a poblaciones generales; aunque hay algunos trabajos sobre población española que muestran variaciones en la prevalencia de SM en función de diferentes ocupaciones laborales ${ }^{16}$. En la actualidad es escasísima la documentación que nos informa sobre la situación en la que se encuentran las poblaciones aeronáuticas ${ }^{17-19}$.

\section{REFERENCIAS BIBLIOGRÁFICAS}

1. Frolich ED, Eric acid: a risk for coronary Herat disease JAMA. 1993; 270:354-359.

2. Niskanen LK, Laaksonen DE, Nyyssonen K, Alfthan G, Lakka HM, Lakka TA, Solonen JT. Uric acid level as a risk factor for cardiovascular and all-cause mortality in middle-aged men. Arch Int Med 2004; 164: 1546-1551. 
3. Tykarski A: Evaluation of renal handling of uric acid in essential hypertension: hyperuricemia related to decreased urate szecretion. Nephron. 1991; 59: 364-368.

4. Expert Panel on the detection, Evaluation and Treatment of High blood cholesterol in Adults: Executive Summary of Third Report of the National cholesterol Education Program (NCEP) Expert Panel on Detection, Evaluation and Treatment of High Blood Cholesterol in Adults (Adult Treatment Panel III). JAMA 2001; 285: 2486- 2497.

5. Ford ES, Mokdad AH, Giles WH. Trends in waist circumference among US adults. Obes Res 2003; 11: 1223-1231.

6. Assman G, Jerzy-Roch N, Schulte H. Cardiovascular risk assessment in metabolic syndrome: View from PROCAM. Endocrinol Metab Clin N Am 2004; 33: 377-392.

7. Einhorn D, Reaven GM, Cobin RH et al. American College of Endocrinology Position Statement on the Insulin Resistance Syndrome. Endocr Pract 2003; 9: 237-252.

8. Ferrannini E, Natali A, Bell P, Cavallo-perin P, Lalic N, Mingrone G. On behalf of the European Group for the Study of the Insulin Resistance. Insulin resistance and hypersecretion in obesity. J Clin Invest 1997; 100: 1166-1173.

9. Centres for Disease Control and Prevention. The third national health and nutrition examination survey (NHANES III) reference manuals and reports: Bethesda Centres for Disease Control and Prevention; 1996.

10. Yamasaki T, Tomita K. Relationship between hyperuricemia and metabolic syndrome. Nippon Rinsho. 2008 Apr ;66(4):766-70.

11. Onat A, Uyarel H, Hergenc G, Karabulut A, Albayrak S, Sari I, Yazici M, Keles I. Serum uric acid is a determinant of metabolic syndrome in a population-based estudy. Am J Hypertens.2006 Oct; 19(10): 1055-62.

12. López-Suárez A, Elvira-González J, Bascuñana-Quirell A, Rosal-Obrador J, Michán-Doña A, EscribanoSerrano J, et al. FERUM urate levels and urinary acid excretion in subjects with metabolic síndrome. Med Clin (Barc) 2006; 126 (9): 321-4.

13. Sánchez-Chaparro, MA, Calvo-Bonacho E, González-Quintela A,Fernández-Labandera C, Cabrera M, Sainz JC, et al: Occupation-related differences in the prevalence of metabolic síndrome. Diabetes Care 2008; 31:1884-1886.

14. Hjortnaes J, Algra A, Olijhoek J, Huisman L, Jacobs J,Van der Graaf Y, Visseren F. Serum uric acid levels and risk for vascular diseases in patients with metabolic síndrome. J Rheumatol. 2007; 34(9):1882-7.

15. Chen LK, Lin MH, Lai HY, Hwang SJ, Chion ST. Uric acid: a surrogate of insulin resistance in older woman. Maturitas. 2008; 59(1): 55-61.

16. Gatti A, Morini E, Cosmo SD, Maiani F, Mandosi E, Fallarino M, et al. Obesity. 2008; 16(4):899-901.

17. Alonso C, Medina J, Gutierrez C. Prevalencia de síndrome metabólico en un colectivo de 762 pilotos de líneas aéreas españolas. Estudio 2003- 2004. Med Aeroesp Ambient 2007; 2:64-71.

18. Khazale NS, Haddad F. Prevalence and characteristics of metabolic syndrome in 111 Royal Jordinian Air Force pilots. Aviat Space Environ Med 2007; 78:968-72.

19. Alonso C, Medina J, Gutiérrez C. Prevalencia de síndrome metabólico en pilotos de líneas aéreas españolas de diferentes grupos de edad. Estudio 2007. Av Diabetol. 2008; 24(4):231-236. 